\title{
Growth performance, duodenal morphology and the caecal microbial population in female broiler chickens fed glycine-fortified low protein diets under heat stress conditions
}

\begin{abstract}
ABSTRCT
This study was undertaken to examine the effect of feeding glycine (Gly)-fortified low protein (LP) diets on the growth performance, duodenal morphology and caecal microbial populations of broiler chickens raised under unheated, cyclic or constant heat stress environmental conditions. 2. From d 1 to 21 (starter phase), an equivalent number of birds were fed either a normal protein (NP) diet or a LP diet fortified with Gly. From d 22 to 42 (grower phase), an equivalent number of birds from each starter diet were distributed to one of the following dietary groups: (i) an NP diet during the starter and grower phases (NPNP), (ii) an NP diet during the starter phase and a LP diet during the grower phase (NPLP), (iii) an LP diet during the starter phase and an NP diet during the grower phase (LPNP) or (iv) LP diets during both phases (LPLP). 3. Commencing from $\mathrm{d} 22$, an equivalent number of birds from each dietary group were exposed to (i) $23 \pm 1^{\circ} \mathrm{C}$ throughout (unheated), (ii) $34 \pm 1^{\circ} \mathrm{C}$ for $7 \mathrm{~h}$ each day from 10:00 to 17:00 (cyclic heat) or (iii) 34 $\pm 1^{\circ} \mathrm{C}$ throughout (constant heat). 4 . Feeding the LP diet during the starter phase resulted in feed intake (FI), weight gain (WG), feed conversion ratios (FCR) and energy efficiency ratios (EER) similar to those for the NP diet. The birds fed the LP diet had a significantly higher protein efficiency ratio (PER) compared with the birds fed the NP diet. 5. During the grower phase, there were significant diet $\times$ temperature interactions for F, WG, FCR, PER, EER, villus height, crypt depth and caecal Clostridia. The birds fed the NPLP and LPLP diets had lower FI, WG and EER, higher FCR, shorter villus height and crypt depth and higher caecal Clostridia compared with the birds fed LPNP and NPNP diets under constant heat stress. However, feeding birds the NPLP and LPLP diets resulted in FI, WG, EER, FCR, morphology parameters and caecal Clostridia equivalent to the birds fed LPNP and NPNP diets, as well as improved PER, under unheated and cyclic heat stress conditions. 6. In conclusion, our results indicate that Gly-fortified LP diets can be fed to broilers under normal and acute heat stress environmental conditions without any adverse effects on performance. However, the use of such LP diets can be detrimental to broilers under chronic heat stress conditions.
\end{abstract}

Keyword: Broilers; Caecal microbiota; Glycine fortification; Heat exposure; Low CP diet; Morphometric indices; Productivity 